\title{
Sex differentiates the role of emotional prosody during word processing
}

\author{
Annett Schirmer*, Sonja A. Kotz, Angela D. Friederici \\ Max Planck Institute of Cognitive Neuroscience, Stephanstrasse 1a, 04103 Leipzig, Germany
}

Accepted 31 January 2002

\begin{abstract}
The meaning of a speech stream is communicated by more than the particular words used by the speaker. For example, speech melody, referred to as prosody, also contributes to meaning. In a cross-modal priming study we investigated the influence of emotional prosody on the processing of visually presented positive and negative target words. The results indicate that emotional prosody modulates word processing and that the time-course of this modulation differs for males and females. Women show behavioural and electrophysiological priming effects already with a small interval between the prosodic prime and the visual target word. In men, however, similar effects of emotional prosody on word processing occur only for a longer interval between prime and target. This indicates that women make an earlier use of emotional prosody during word processing as compared to men. (C) 2002 Elsevier Science B.V. All rights reserved.
\end{abstract}

Theme: Neural basis of behaviour

Topic: Cognition

Keywords: Emotional prosody; Sex differences; N400; Semantic priming

\section{Introduction}

Context in the word processing literature is usually conceived of as the semantic structure established by the words in a sentence $[8,23]$. That semantic structure or context facilitates word processing has been shown in numerous priming studies $[18,22]$. In these studies, faster reaction times and higher accuracy rates were obtained for target words that matched the preceding sentence context as compared to target words that did not match. Eventrelated potentials (ERPs) are also sensitive to the effects of context during word processing. For example, the amplitude of the N400 component, a negative going waveform that peaks around $400 \mathrm{~ms}$ following word onset, is smaller when a word matches the preceding semantic context as compared to when it does not [14].

Context, however, is not limited to semantics. In everyday communication, non-verbal cues, such as emotional prosody, reveal additional sources of information [3,4]. For 0113 .

*Corresponding author. Tel.: +49-341-994-0157; fax: +49-341-994-

E-mail address: schirmer@cns.mpg.de (A. Schirmer). example, a verbal message such as 'That was a good idea!' spoken with an angry voice is unlikely to be a compliment. Nevertheless, the time-course of the interaction between emotional prosody and word meaning has not been investigated to date. If emotional-prosodic context modulates word processing in a similar fashion as semantic context, then it should elicit priming effects comparable to those obtained for semantic priming. Because women are reported to be more emotionally expressive $[6,20]$ than men, as well as better able to understand emotional expression from faces, gestures and voices [10], we were concerned that women would differ from men in their reliance on emotional-prosodic cues. To address this possibility, we included sex as a factor in our data analyses.

To establish emotional-prosodic priming in men and women we conducted a cross-modal priming study. Primes were semantically neutral German sentences spoken with either a happy or a sad intonation (e.g., 'Yesterday she had her final exam.'). Following each prime a visual target appeared in the centre of the computer screen in front of the subject. The target was either a German word or a pseudoword. Legal target words were semantically related to the sentence final word. For half of these legal words, 
word valence and prime prosody matched (e.g., 'success' following a happy intonation) whereas for the remaining words, word valence and prime prosody did not match (e.g., 'failure' following a happy intonation). Participants indicated whether the target was a word or a pseudoword by pressing one of two buttons. Furthermore, subjects were told that the auditory primes were not relevant to the task and that they should just listen to them.

\section{Experiment 1}

\subsection{Methods}

Thirty-two volunteers participated in Experiment 1. Sixteen participants were female with a mean age of 23.81 (S.D.=3.01). The remaining 16 male participants had a mean age of 24 (S.D.=2.03). All subjects were righthanded, native speakers of German, had normal or corrected to normal vision and no hearing impairments.

The stimulus material consisted of 99 sentences, of which 49 were experimentally relevant and 50 were fillers. A female native speaker of standard German produced all sentences with a happy and sad intonation resulting in a total of 198 sentences. Sentences were taped with a DAT recorder and digitised at a $16-\mathrm{bit} / 44.1-\mathrm{kHz}$ sampling rate. For all experimentally relevant sentences two corresponding words were selected on the basis of a prior association study. One word had a positive meaning and the other word a negative meaning. Both groups of target words did not differ in word frequency (Celex [2]) and word length. A prior rating with a different group of subjects ensured that positive and negative words differed significantly in valence but that absolute valence strength was identical (i.e. positive words were rated as positive as negative words were rated negative). For 20 out of the 50 filler sentences, an affectively valenced filler word as well as a pseudoword was selected. In a given experimental session, half the filler words matched the prosodic valence of the preceding filler sentence and half did not. For each of the remaining 30 filler sentences two pseudowords were selected. Each sentence was presented twice with a positive prosody and twice with a negative prosody. The target word following the sentence had either the same or a different valence as the sentence prosody. This sums up to four experimental conditions: match and mismatch condition for positive target words as well as match and mismatch condition for negative target words. Happy and sad filler sentences were also presented twice followed by either a happy or a sad word or a pseudoword, respectively. To avoid repetition effects, the stimulus material was divided so that a given target was presented only once to each subject. Consequently, every subject saw 98 experimentally relevant target words and 100 fillers. Trials were pseudo-randomised and distributed over four blocks.
The interstimulus interval (ISI) between prime and target was $200 \mathrm{~ms}$.

Testing was carried out in a soundproof and electrically shielded chamber. Subjects were seated in a comfortable chair facing a computer monitor at a distance of $1.15 \mathrm{~m}$. In order to familiarise participants with the task, each experimental session started with a block of eight practice trials. Half the subjects responded to words with their right hand and to pseudowords with their left hand. For the remaining subjects the response pattern was reversed.

The electroencephalogram (EEG) was recorded $(250 \mathrm{~Hz}$ sampling rate) from 58 electrodes, which were placed on the head according to the modified expanded 10-20 system. The reference electrode was placed on the tip of the nose. In order to control for horizontal and vertical eye movements, a bipolar electrooculogram was recorded using four electrodes. Electrode resistance was kept below $5 \mathrm{kOhm}$. ERP averages were computed with a $150 \mathrm{~ms}$ prestimulus baseline and a $1000 \mathrm{~ms}$ ERP time window. Trials containing eye blinks or movement artefacts were omitted from the ERP analysis.

For statistical analysis, electrodes were grouped into four regions of interest (Fig. 1): left anterior (F7, F5, F3, FT7, FC5, FC3, T7, C5, C3), right anterior (F8, F6, F4, FT8, FC6, FC4, T8, C6, C4), left posterior (TP7, CP5, $\mathrm{CP} 3, \mathrm{P} 7, \mathrm{P} 5, \mathrm{P} 3, \mathrm{PO} 7, \mathrm{PO} 3, \mathrm{O} 1)$ and right posterior (TP8, CP6, CP4, P8, P6, P4, PO8, PO4, O2). ERPs measured at anterior and posterior regions of interest (ROIs) established the factor Anterior/Posterior in the statistical analysis and ERPs measured at right and left hemisphere ROIs estab-

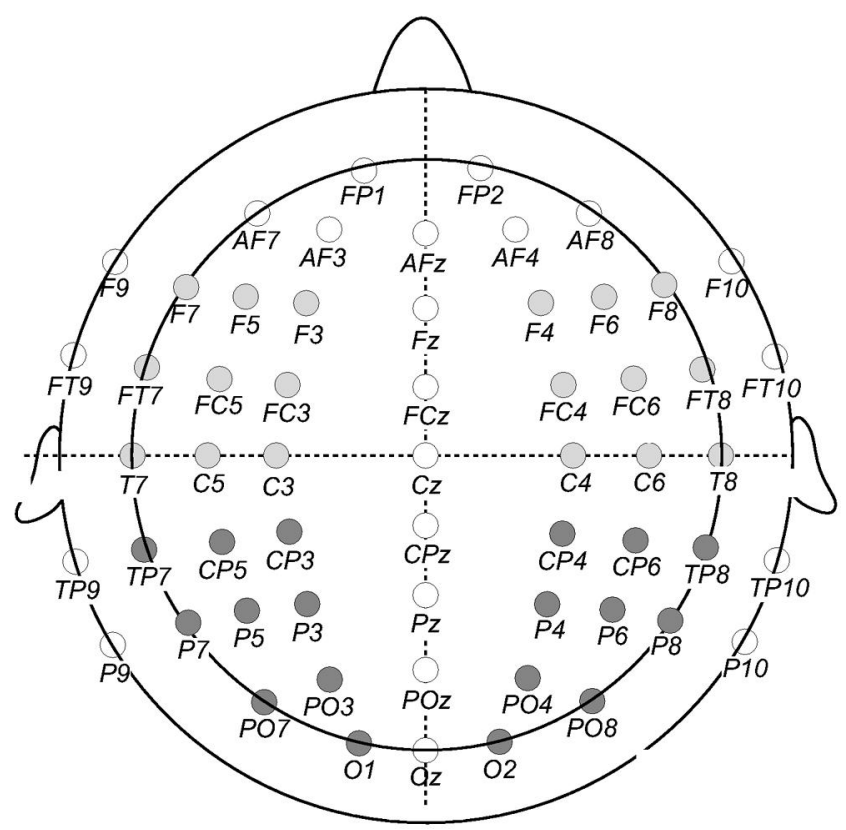

Fig. 1. Open circles indicate electrodes that were recorded but did not enter statistical analysis. Circles filled with light grey indicate electrodes that entered right and left anterior regions of interest. Circles filled with dark grey indicate electrodes that entered right and left posterior regions of interest. 
lished the factor Hemisphere. ERPs were quantified as mean amplitudes in three different time windows in each of the ROIs. Three separate ANOVAS were conducted for the following three time windows: the P200 (150-300 ms), the N400 (300-550 ms), and the P300 (550-700 ms). Match (match/mismatch), Target (positive/negative), Anterior/Posterior (anterior/posterior) and Hemisphere (left/ right) were repeated factors and Sex was a betweensubjects factor. Significant interactions are reported only when post hoc comparisons revealed significant effects. For illustration only, grand averages were smoothed with an $8 \mathrm{~Hz}$ low-pass filter.

\subsection{Results}

A Sex by Match interaction in the behavioural $\left(F_{1,30}=\right.$ $4.65, P<0.05)$ as well as in the electrophysiological data (P200, $F_{1,30}=4.18, P<0.05 ; \mathrm{N} 400, F_{1,30}=4.27, P<0.05$; $\left.\mathrm{P} 300, F_{1,30}=8.0, P<0.01\right)$ indicated processing differences between men and women. Women responded faster to target words that matched the valence of the preceding prime's prosody compared to non-matching words $\left(F_{1,15}=\right.$ 6.45, $P<0.05$; Fig. 2b). Additionally, female subjects showed a smaller N400 amplitude for the match as compared to the mismatch condition $\left(F_{1,15}=19.42, P<\right.$ 0.001; Fig. 3). This emotional-prosodic priming effect already started to differentiate in a preceding ERP component, the $\mathrm{P} 200\left(F_{1,15}=7.25, P<0.05\right)$, and was still present in the later P300 $\left(F_{1,15}=7.64, P<0.05\right)$. Consequently, the match conditions differed significantly from the mismatch conditions between 150 and $700 \mathrm{~ms}$ follow-
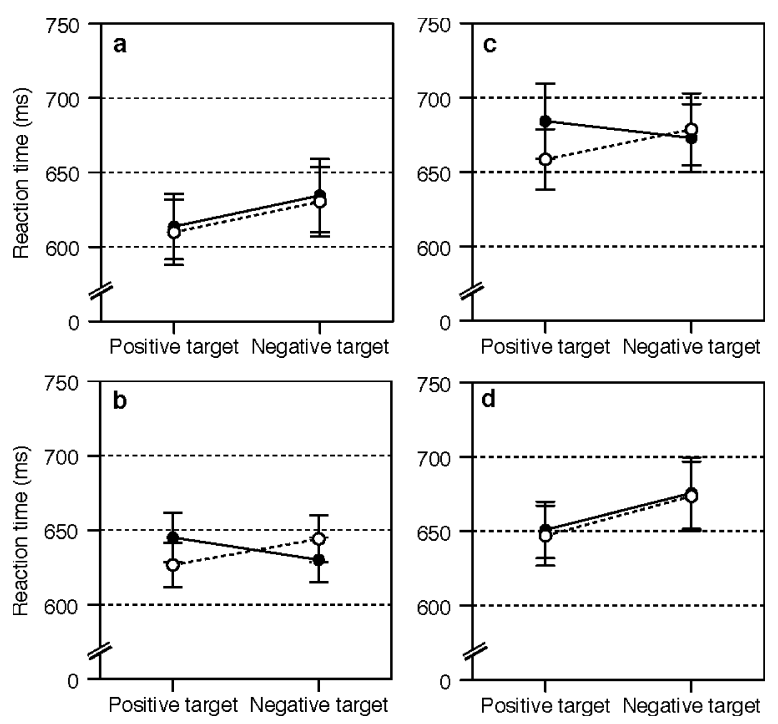

Fig. 2. Mean reaction times ( \pm 1 S.E.M.) for lexical decisions plotted as a function of target valence. Open circles together with dotted lines indicate a positive prime prosody, closed circles together with solid lines indicate a negative prime prosody. Experiment 1 (ISI $200 \mathrm{~ms}$ ) is presented in (a), male subjects and (b), female subjects. Experiment 2 (ISI $750 \mathrm{~ms}$ ) is presented in (c), male subjects and (d), female subjects.
Male subjects

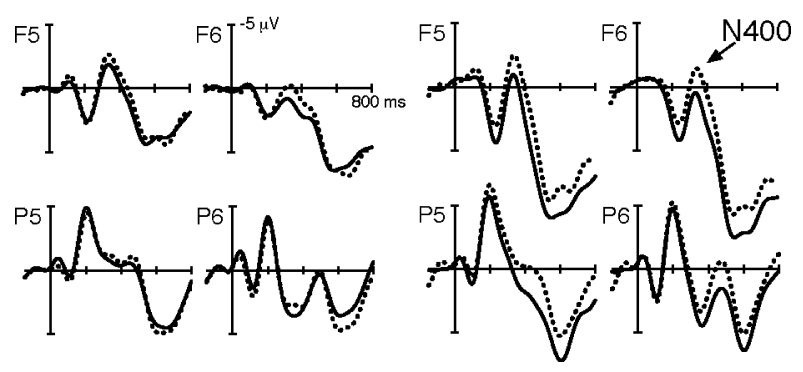

Fig. 3. ERPs time-locked to target onset for experiment 1 (ISI $200 \mathrm{~ms}$ ). A mismatch between prime and target (dotted line) elicited a larger N400 response compared to a match (solid line) only in female subjects. This priming effect started to differentiate in the P200 and was also present in the P300.

ing word onset. Furthermore, this effect was broadly distributed over the scalp since the Match by Sex interaction was not modified by either Hemisphere or Anterior/ Posterior $\left(F_{1,30}<1\right)$.

Male subjects failed to demonstrate the expected behavioural and electrophysiological emotional-prosodic priming effects $\left(F_{1,15}<1\right)$. Moreover, a significant Sex by Target interaction in the reaction time data $\left(F_{1,30}=10.37\right.$, $P<0.01)$ indicated different processing for negative and positive words in men but not in women. Male participants showed faster reaction times to positive target words as compared to negative target words independent of the prime's prosody $\left(F_{1,15}=25.27, P<0.001\right.$; Fig. 2a). A statistical analysis of the accuracy data revealed no significant effects. Across conditions, participants' accuracy scores were above $97 \%$.

In sum, these results suggest that shortly after the prime, women and men process the target word in very different ways. While women clearly use the prosodic context during the processing of the target word, men process the target word with regard to word valence, but completely independent of prosodic information.

To determine whether prosodic information differentiates matching from non-matching targets in men at a later point in time, a second experiment with a longer ISI (750 ms) was conducted.

\section{Experiment 2}

\subsection{Methods}

Sixteen males and 16 females that did not participate in Experiment 1 were recruited for Experiment 2. Female participants had a mean age of 21.87 (S.D. $=1.92$ ) and male participants had a mean age of 23.37 (S.D.=3.03). All subjects were right-handed, native speakers of German, had normal or corrected-to-normal vision and no hearing impairments. 
Stimulus material and procedure were comparable to Experiment 1, with the exception that the stimulus interval between prime and target was now $750 \mathrm{~ms}$.

\subsection{Results}

Also with the longer ISI, ERPs revealed a significant Sex by Match interaction (N400, $F_{1,30}=7.87, P<0.01$ ). Post hoc comparisons indicated that women no longer show emotional-prosodic priming $\left(F_{1,15}<1\right)$. Also the reaction times revealed no significant priming in women. The Match by Sex interaction was non-significant $\left(F_{1,30}=\right.$ 2.59, $P=0.117)$ and the Match main effect showed only as a tendency $\left(F_{1,30}=3.29, P=0.079\right)$. Instead, women's behavioural response to the target words was similar to what was found for men in the previous experiment. Women responded faster to positive as compared to negative target words independent of the prime's prosody (Sex by Target, $F_{1,30}=6.81, P<0.05$; females, $F_{1,15}=$ 16.84, $P<0.001$; males, $F_{1,15}<1$ ).

In males, reaction times no longer differentiated between positive and negative target words (Fig. 2d). Instead, ERPs indicated significant emotional-prosodic priming. Emotional congruence between prime and target elicited a smaller $\mathrm{N} 400$ as compared to incongruence $\left(F_{1,15}=6.07, P<0.05\right.$; Fig. 4). In contrast to the priming effect reported for women in the previous experiment, the priming effect in men was restricted to the typical N400 time range between 300 and $550 \mathrm{~ms}$ following word onset. The lack of an interaction with the factors Anterior/ Posterior and Hemisphere $\left(F_{1,30}<1\right)$ indicated that the N400 effect was broadly distributed over the scalp. Again, no effects were found for accuracy. Subjects performed equally well across conditions.

Taken together, the second experiment supports the notion of processing differences in men and women. With the longer ISI, word processing in male subjects profits to some extent from the given emotional-prosodic context.
Male subjects
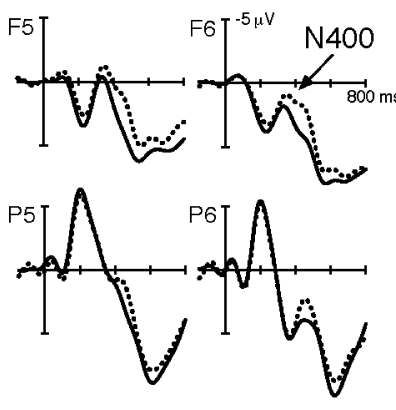

Female subjects
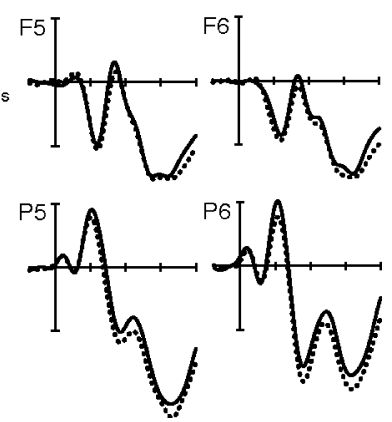

Fig. 4. ERPs time locked to target onset for experiment 2 (ISI $750 \mathrm{~ms}$ ). A mismatch between prime and target (dotted line) elicited a larger N400 response compared to a match (solid line) only in male subjects.
However, there was no behavioural priming and the priming effect in the ERP was restricted to the N400 time range. Moreover, word processing in females was no longer influenced by the emotional-prosodic context but solely by word valence. This reduction in priming could be due to the fact that with a longer ISI processing is less automatic and more influenced by strategies [18].

Because men and women may have experienced the emotional-prosodic conditions in different ways, an expectancy judgement followed every experimental session. In the expectancy judgement, as in the priming experiments, subjects heard a sentence and then saw a corresponding target word on the computer screen. Subjects were asked to judge on a five-point scale as to how expected or unexpected the target word given the preceding sentence was. The subject's judgement entered an ANOVA with Target (positive/negative) and Match (match/mismatch) as repeated factors and ISI (200/750) as well as Sex as between subject factors. Statistical analyses indicated no difference concerning this judgement between male and female subjects $\left(F_{1,61}<1\right)$ as well as between the first and the second experiment $\left(F_{1,61}<1\right)$. However, there was a significant Target main effect indicating that target expectancy was higher for positive as compared to negative targets $\left(F_{1,61}=119.30, P<0.0001\right.$; Fig. 5). This valence effect is in accordance with earlier findings of negative events being less expected and capturing more attention $[5,16]$. Additionally, a match between the target valence and prime's prosody was experienced as more expected than a mismatch $\left(F_{1,61}=149.69, \quad P<\right.$ $0.0001)$.
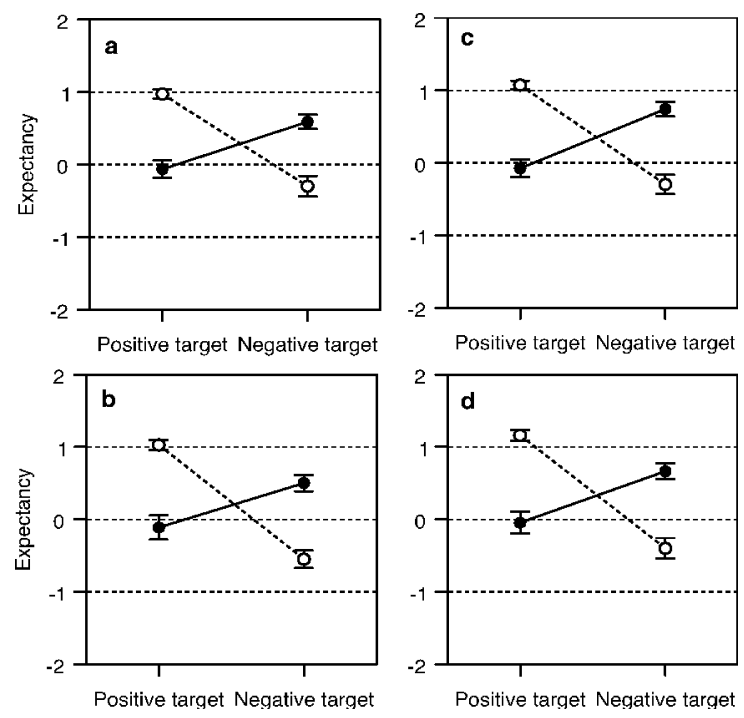

Fig. 5. Mean congruence ( \pm 1 S.E.M.) for the congruence judgement plotted as a function of target valence. Open circles together with dotted lines indicate a positive prime prosody, closed circles together with solid lines indicate a negative prime prosody. Experiment 1 (ISI $200 \mathrm{~ms}$ ) is presented in (a), male subjects and (b), female subjects. Experiment 2 (ISI $750 \mathrm{~ms}$ ) is presented in (c), male subjects and (d), female subjects. 


\section{Dichotic listening}

Meta-analyses, summarising several dichotic listening studies indicated that women are less lateralised than man when processing language [11,17]. Because this could explain the temporal advantage of women in our data, all subjects were reinvited to participate in a dichotic listening experiment. Fifty-five of the 64 ERP participants returned for the dichotic listening study. One woman and three men were missing from Experiment 1; two women and three men were missing from Experiment 2. The subjects listened over headphones, first monaurally and then binaurally, to a series of rhyme words (e.g., bank and tank). Their task was to chose from a set of four words presented on the computer screen the one they thought they had just heard. In the case of binaural presentation, they had to report the more dominant word. For each subject a Lambda coefficient was computed, which entered an ANOVA with Sex and Experiment (ISI 200/ISI 750) as between-subject factors. The results indicate that male and female subjects did not differ significantly in language lateralisation $\left(F_{1,51}<1\right)$.

\section{Discussion}

In two cross-modal priming experiments, we found evidence for the influence of emotional prosody on word processing. Words that were congruent in valence to the emotional-prosodic context were responded to faster and elicited a smaller N400 amplitude as compared to incongruent words. In the word processing literature, the N400 component is thought to reflect the integration of a word in the preceding semantic context [7]. A variety of ERP studies demonstrated that the stronger the semantic context and the easier the integration of a word the smaller is the N400 amplitude elicited by the word $[14,13]$. The present findings indicate a similar sensitivity of the N400 component to emotional-prosodic context. Therefore, we argue that emotional prosody guides the contextual integration of a word similar to semantics.

Although present in both men and women, the timecourse of this integration process differs as a function of sex. In women, the behavioural and ERP data indicate an interaction between word meaning and emotional-prosodic context with a short ISI (200 ms). Furthermore, the N400 effect in the ERP starts no later than $150 \mathrm{~ms}$ following word onset. In men, the behavioural and ERP data for the short ISI (200 ms) reflect context independent processing of the word valence. An N400 effect indicating emotionalprosodic priming is evident only with the longer ISI (750 $\mathrm{ms})$. Moreover, this effect begins to differentiate match and mismatch condition $300 \mathrm{~ms}$ following word onset, which is $150 \mathrm{~ms}$ later than the N400 effect found in women.

Our results clearly indicate that, compared to men, women make a faster use of emotional prosody during language processing. This advantage might arise from differences in the processing as well as the organisation of language in the brain. For example, multiple findings from fMRI [21], dichotic listening [11] and visual laterality studies [12] suggest stronger lateralisation of language functions to the left hemisphere in males, whereas language functions in females tend to be organised more bilaterally. Because the processing of emotional prosody is thought to be lateralised to the right hemisphere [19], women might profit from shorter neuronal connections between areas that process language and areas that process emotional prosody. The dichotic listening test in our subjects, however, failed to reveal even a tendency toward a sex difference in language lateralisation.

Besides lateralisation, interhemispheric connectivity could be responsible for the faster interaction between emotional prosody and word information in women. Brain structures connecting both hemispheres, such as the anterior commissure, the massa intermedia and the splenium of the corpus callosum are somewhat larger in women than in men $[1,15]$. One might assume that greater interconnectivity facilitates the transfer of information between the two hemispheres. However, interhemispheric transmission time has been found to be shorter in men than in women [9]. Therefore, reasons other than lateralisation and connectivity might contribute to the present findings. For example, language processing itself may differ between men and women so that depending on sex the different types of information play a different role throughout language processing. Given the faster integration of emotional prosody and word information in women we would like to argue that, compared to men, women more automatically rely on emotional-prosodic context when processing language.

In contrast to previous studies $[6,10,20]$ of sex differences in emotional tasks, our results do not suggest that women are simply 'more emotional'. In fact, both men and women show strong valence effects. Furthermore, when asked to judge target expectancy both sexes use the prosodic context to guide their judgement. Women, however, profit very early from emotional-prosodic cues when processing words while men initially focus solely on word valence. Moreover, this time difference suggests that, the combined processing of emotions from words and prosody might be more efficient in women as compared to men. Given that in connected speech the duration of a twosyllabic word seldom exceeds $600 \mathrm{~ms}$ with the next word immediately following, a processing difference of a few hundred milliseconds is likely to be of functional importance. However, this female advantage may only apply to situations in which emotions are expressed on a verbal and a prosodic level but not for verbal processing alone.

The present findings establish for the first time that under certain circumstances speech processing in men and women can be different. At the same time these findings give rise to other questions such as whether there are sex 
differences in the expression of emotions. For example, men might preferably express emotions verbally rather than prosodically whereas the reversed might be true for women. What causes sex differences in the processing of emotional speech and in what way do they affect understanding? These and other questions need to be addressed in future research.

\section{Acknowledgements}

We would like to thank T. Gunter, T. Penney, C. Kranczioch, C. Friedrich and M. De Filippis for discussion on the design and comments on the manuscript. This work was supported by the Leibniz Science Prize awarded to A.D. Friederici and the German Research Foundation DFG.

\section{References}

[1] L.S. Allen, R.A. Gorski, Sexual orientation and the size of the anterior commissure in the human brain, Proc. Natl. Acad. Sci. USA 89 (1992) 7199-7202.

[2] R.H. Baayen, R. Piepenbrock, H. van Rijn, The CELEX Lexical Database, Center for Lexical Information, MPI for Psycholinguistics, Nijmegen, 1995, [CD-ROM].

[3] K.M. Baum, S. Nowicki, Perception of emotion: measuring decoding accuracy of adult prosodic cues varying in intensity, J. Nonverb. Behav. 22 (1998) 89-107.

[4] L.X. Blonder, D. Bowers, K.M. Heilman, The role of the right hemisphere in emotional communication, Brain 114 (1991) 11151127.

[5] B.P. Bradley, K. Mogg, N.H. Millar, Covert and overt orienting of attention to emotional faces in anxiety, Cogn. Emot. 14 (2000) 789-808.

[6] K. Brennan-Parks, M. Goddard, A.E. Wilson, L. Kinnear, Sex differences in smiling as measured in a picture taking task, Sex Roles 24 (1991) 375-382.

[7] D.J. Chwilla, C.M. Brown, P. Hagoort, The N400 as a function of the level of processing, Psychophysiology 32 (1995) 274-285.

[8] C. Connine, Effects of sentence context and lexical knowledge in speech processing, in: G.T.S. Altmann (Ed.), Cognitive Models of
Speech Processing: Psycholinguistic and Computational Perspectives, MIT Press, Cambridge, MA, 1990, pp. 281-294.

[9] G. Geffen, R. Virginia, M. Luciano, Sex differences in the perception of tactile simultaneity, Cortex 36 (2000) 323-335.

[10] J.A. Hall, Gender effects in decoding nonverbal cues, Psychol. Bull. 85 (1978) 845-857.

[11] M. Hiscock, R. Inch, C. Jacek, C. Hiscock-Kalil, K.M. Kalil, Is there a sex difference in human laterality? I. An exhaustive survey of auditory laterality studies from six neuropsychological journals, J. Clin. Exp. Neuropsychol. 16 (1994) 423-435.

[12] M. Hiscock, M. Israelian, R. Inch, C. Jacek, C. Hiscock-Kalil, Is there a sex difference in human laterality? II. An exhaustive survey of visual laterality studies from six neuropsychology journals, J. Clin. Exp. Neuropsychol. 17 (1995) 590-610.

[13] P.J. Holcomb, J.E. Anderson, Cross-modal semantic priming: a time course analysis using event-related brain potentials, Lang. Cogn. Proc. 8 (1993) 379-411.

[14] M. Kutas, S.A. Hillyard, Reading senseless sentences: brain potentials reflect semantic incongruity, Science 207 (1980) 203-205.

[15] M.C. De Lacoste-Utamsing, R.L. Holloway, Sexual dimorphism in the human corpus callosum, Science 216 (1982) 1431-1432.

[16] S.F. Lang, C.A. Nelson, P.F. Collins, Event-related potentials to emotional and neutral stimuli, J. Clin. Exp. Neuropsychol. 12 (1990) 946-958.

[17] J. McGlone, Sex differences in human brain asymmetry: a critical survey, Behav. Brain Sci. 3 (1980) 215-263.

[18] H.N. Neely, Semantic priming effects in visual word recognition: a selective review of current findings and theories, in: D. Besner, G.W. Humphreys (Eds.), Basic Processes in Reading: Visual Word Recognition, Erlbaum, Hilsdale, NJ, 1991, pp. 264-336.

[19] M.D. Pell, S.R. Baum, The ability to perceive and comprehend intonation in linguistic and affective contexts by brain-damaged adults, Brain Lang. 57 (1997) 80-99.

[20] M. Polce-Lynch, B.J. Myers, C.T. Kilmartin, R. Forssmann-Falck, W. Kliewer, Gender and age patterns in emotional expression, body image, and self-esteem: a qualitative analysis, Sex Roles 38 (1998) 1025-1048.

[21] B.A. Shaywitz, S.E. Shaywitz, K.R. Pugh, R.T. Constable, P. Skudlarski, R.K. Fulbright, R.A. Bronen, J.M. Fletcher, D.P. Shankwiler, L. Katz, J.C. Gore, Sex differences in the functional organization of the brain for language, Nature 373 (1995) 607-609.

[22] D.A. Swinney, Lexical access during sentence comprehension: (re)consideration of context effects, J. Verb. Learn. Behav. 18 (1979) 645-659.

[23] P. Zwitserlood, Spoken words in sentence contexts, in: A.D. Friederici (Ed.), Language Comprehension: A Biological Perspective, Springer, Berlin, 1999, pp. 71-100. 